\title{
Lung Ultrasound in the Emergency Department for Early Identification of COVID-19 Pneumonia
}

\author{
Alessandro Zanforlina,b Giacomo Strapazzon ${ }^{c, d}$ Markus Falk ${ }^{c}$ \\ Valentina Gallina ${ }^{\mathrm{e}}$ Antonio Viteritti ${ }^{\mathrm{e}}$ Laura Valzolgher ${ }^{\mathrm{e}}$ Mario La Guardia ${ }^{\mathrm{e}}$ \\ Federica Ferro $^{a}$ Leonardo Pagani ${ }^{f}$ Norberto Vezzali ${ }^{a}$

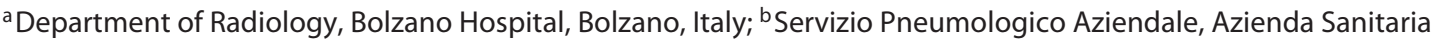 \\ dell'Alto Adige, Bolzano, Italy; ' Institute of Mountain Emergency Medicine, Eurac Research, Bolzano, Italy; \\ 'Department of Anesthesiology and Critical Care Medicine, Medical University of Innsbruck, Innsbruck, Austria; \\ e Department of Emergency Medicine, Bolzano Hospital, Bolzano, Italy; ${ }^{\mathrm{f}}$ Infectious Disease Unit, Bolzano Hospital, \\ Bolzano, Italy
}

\section{Keywords}

Lung ultrasound $\cdot$ COVID-19 $\cdot$ Pneumonia diagnostics · Viral infection · Emergency department

\begin{abstract}
Background: Coronavirus disease 2019 (COVID-19) is a pandemic overwhelming the health care systems worldwide. Lung ultrasound (LUS) use has been proposed to identify suspected COVID-19 patients and direct them to the isolation area in the emergency department (ED) or to discharge them for outpatient treatment. Objective: Our aim was to retrospectively investigate the use of LUS in the ED to identify COVID-19 pneumonia (CP). Methods: We performed a retrospective single-center study including all patients accessing the ED who underwent LUS examination for suspicion of COVID-19 during the initial outbreak. Demographics, clinical parameters, laboratory values, imaging features, and outcome variables were collected. The receiver operating characteristic (ROC) curve was used to evaluate diagnostic accuracy. Results: A total of $41 \%$ patients were COVID19 -positive; $67 \%$ of them were diagnosed with CP. The ROC curve of the LUS score showed an area under the curve of
\end{abstract}

0.837 (95\% Cl 0.75-0.92) and with a cutoff value $\geq 3$ identified 28 of 31 patients with CP and 11 of 15 without (sensitivity 90\%, 95\% Cl 74-97\%; specificity 75\%, 95\% CI 56-76\%). LUS in combination with nasopharyngeal swab has a sensitivity of $100 \%(95 \% \mathrm{Cl} 74-97 \%)$ and a specificity of $61 \%(95 \% \mathrm{Cl}$ 44-67\%). Conclusions: LUS is a promising technique for early identification of CP in patients who accessed the ED in an active epidemic time. The LUS score shows a sensitivity of $90 \%$ for CP, allowing to quickly direct patients with COVID-19 to the ED isolation area or to discharge them for outpatient treatment.

๑) 2020 S. Karger AG, Basel

\section{Introduction}

Coronavirus disease 2019 (COVID-19) is a pandemic overwhelming the health care systems worldwide [1]. Since the beginning of March 2020, emergency departments (EDs) in South Tyrol, Italy had to address an ex-

Study location: Department of Emergency Medicine, Bolzano Hospital, Bolzano, Italy. karger@karger.com www.karger.com/res

(C) 2020 S. Karger AG, Base

Karger"
Giacomo Strapazzon

Institute of Mountain Emergency Medicine

Eurac Research, Viale Druso 1

IT-39100 Bolzano (Italy)

giacomo.strapazzon@eurac.edu 
pected increasing wave of suspected COVID-19 patients to the $\mathrm{ED}$, and different management and triage strategies were undertaken. History of exposure, clinical and laboratory characteristics, imaging tests including chest X-ray, computed tomography (CT), and lung ultrasound (LUS), and real-time polymerase chain reaction (RT-PCR) assay from specimens [2] have guided the emergency provider decision process of suspected COVID-19 patients.

The clinical presentation of COVID-19 ranges from completely asymptomatic cases to very severe pneumonia showing diffuse alveolar damage and airway inflammation [3], acute respiratory distress syndrome, and multiorgan failure. COVID-19 pneumonia (CP) showed a typical CT pattern involving peripheral areas of the lung [4]. Such changes on CT have been compared to LUS findings, showing an encouraging correlation [5-7]. Thanks to the broad diffusion of the technique in Italy [8], the potential role of LUS for early identification of CP was initially proposed by the colleagues in Lodi (Italy) via social media [9]. LUS shows a sensitivity and specificity of $85-93 \%$ and $86-$ $93 \%$, respectively in the diagnosis of pneumonia [10-14]. $\mathrm{Lu}$ et al. [15] described a sensitivity of $69-100 \%$ and a specificity of $86-93 \%$ of LUS in the diagnosis of CP. Compared to CT, LUS is safer, repeatable, radiation-free, cheap, and allows a point-of-care use. Soldati et al. [16] proposed an international standard LUS score for COVID-19 patients. Various perspective papers [17-19] and video tutorials [20] further contributed to theorize report and encouraged its use in the COVID-19 outbreak. Based on the difficulties to perform a high number of CT scans to diagnose $\mathrm{CP}$ due to the lack of resources and dedicated areas [21], we developed a specific protocol including LUS in the evaluation of patients in the ED.

No data were available about the sensitivity and specificity of LUS for early identification of suspected CP in the ED during an active epidemic. Our aim was to retrospectively investigate the use of LUS in the ED to identify CP. Our expectation was that LUS would be able to identify CP with a high sensitivity, and its use among ED management strategies could lead to early diagnosis and management of $\mathrm{CP}$, avoiding unnecessary hospital admissions and enabling EDs to allocate resources more appropriately.

\section{Methods}

Study Population, Setting, and Data Collection

This retrospective single-center study included data of all suspected COVID-19 patients who underwent LUS examination in ED triage at the Regional Hospital of Bolzano, Italy between March
24 and April 14, 2020. Demographics, clinical parameters, laboratory values, imaging features, and outcome variables were collected, and researchers analyzed only anonymized data. This study was registered in the EudraCT database (No. 2020-001785-11).

\section{Case Definition}

Patients were defined as suspect for COVID-19 when presenting two or more of the following criteria: fever, cough, dyspnea, previous contact with positive cases within the last 14 days, or coming from or having been in at-risk areas. A confirmed case of $\mathrm{CP}$ was defined as follows: a patient with suggestive symptoms, with imaging features of pneumonia and positive RT-PCR assay, or one with suggestive symptoms, with or without arterial blood gas (ABG) impairment, with imaging features suggestive of $\mathrm{CP}$ even after a first negative RT-PCR nasopharyngeal swab after consulting with infectious disease consultant, in agreement with the WHO case definition statement [22].

\section{Laboratory Examinations}

Clinical specimens for COVID-19 diagnostic testing were obtained from nasopharyngeal swab and processed by RT-PCR assay, according to the guidelines of the National Institute of Health Care, Italy [23].

Blood samples were obtained for analysis of ABG and routine laboratory, including blood count, lactate dehydrogenase, D-dimer, and C-reactive protein. Additional tests were performed based on clinical course, including $\mathrm{N}$-terminal prohormone of brain natriuretic peptide, procalcitonin, and troponin-I.

\section{Radiologic Procedures}

Chest X-ray was performed with patients in semi-recumbent position and, if made possible by the patient's conditions, orthostatic using analogic portable machines (Mobilett XP Hybrid, Siemens, Erlangen, Germany) and digital machines (Alara DR Max, Siemens; GM85, Samsung, Korea).

CT scan was performed with Somatom Definition Flash and Somatom Definition Edge (Siemens). High-resolution CT was performed with protocol of acquisition without intravenous contrast medium, at the end of inspiration and at the end of expiration, with craniocaudal acquisition; the system of automated modulation of exposition (CAREDose4D) was active. One-millimeter slices were reconstructed on the axial plane with a parenchymal window and $3-\mathrm{mm}$ slices with a mediastinal window, followed by 3-mm multiplanar reconstructions in the coronal and sagittal plane.

\section{LUS Scan}

Ultrasound examinations were performed by four experienced operators using a portable ultrasound device (M-Turbo, Sonosite Fujifilm Europe, Amsterdam, The Netherlands) with a convex transducer (5-2 MHz). After abdominal preset selection (no compound; filters off; general frequency setting), focus was set on the pleural line, depth at $6 \mathrm{~cm}$, and low gain (i.e., to evaluate pleural gliding). The ultrasound device was protected with a plastic film; the probe was covered by a single-use film and underwent full disinfection after every use.

Patients were examined in a sitting position (when possible) with continuous scan in all intercostal spaces, covering the whole chest surface. Based on Bouhemad et al. [24], LUS features were reported in a standard sheet that included ten fields for each lung
Zanforlin et al. 


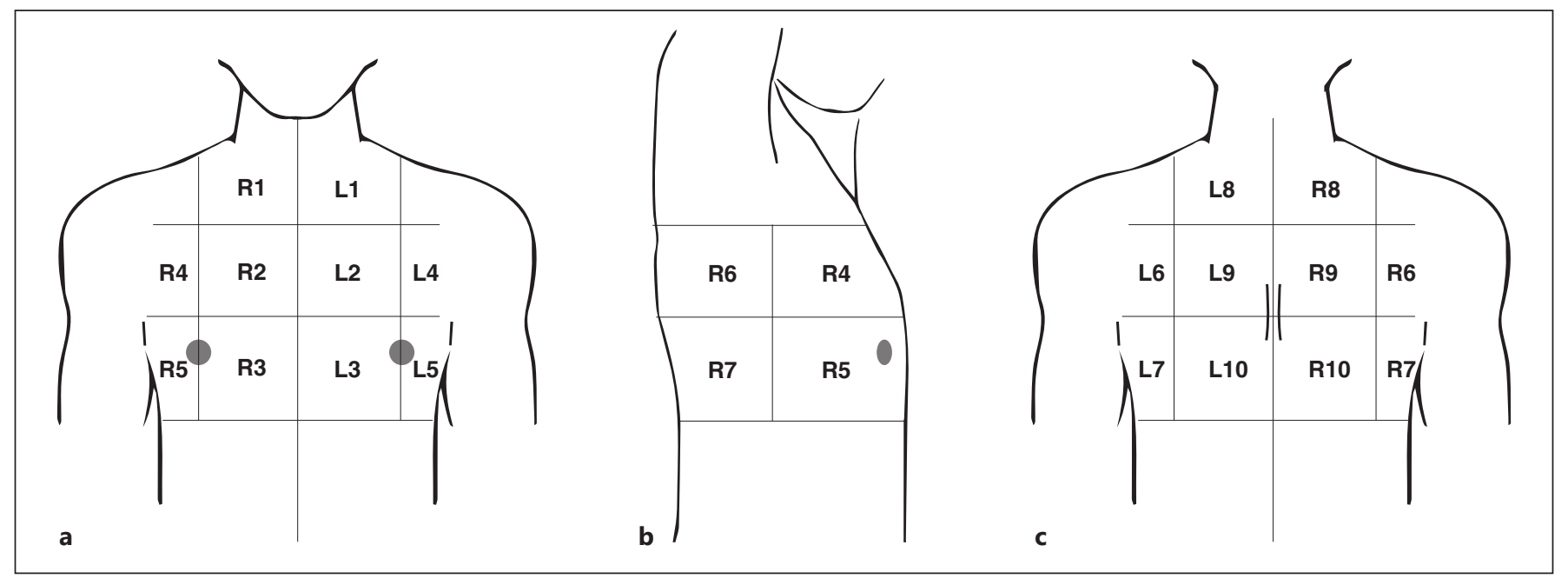

Fig. 1. Chest areas for LUS reporting. The areas allow the mapping of lung lesions to provide a panoramic vision and evaluation. For every area the operator will notice the highest score found. Each lung is divided into 10 areas. a Three parasternal ( 1 apical $=$ apical segment of upper lobe; 2 medium = anterior segment of upper lobe; 3 lower $=$ medial segment of medium lobe/lingular segment) for each side. $\mathbf{b}$ Two axillary anterior $(4$ upper $=$ anterior segment of upper lobe; 5 lower = lateral segment of medium lobe), and two axillary posterior $(6$ upper $=$ apical segment of lower lobe; 7 lower $=$ posterior-lateral basal segments of lower lobe). c Three paravertebral $(8$ upper $=$ posterior segment of upper lobe; 9 medium $=$ apical segment of lower lobe; 10 lower $=$ posterior-lateral basal segments of lower lobe). Areas are divided as right (R) or left (L) based on the side of the patient. LUS, lung ultrasound.
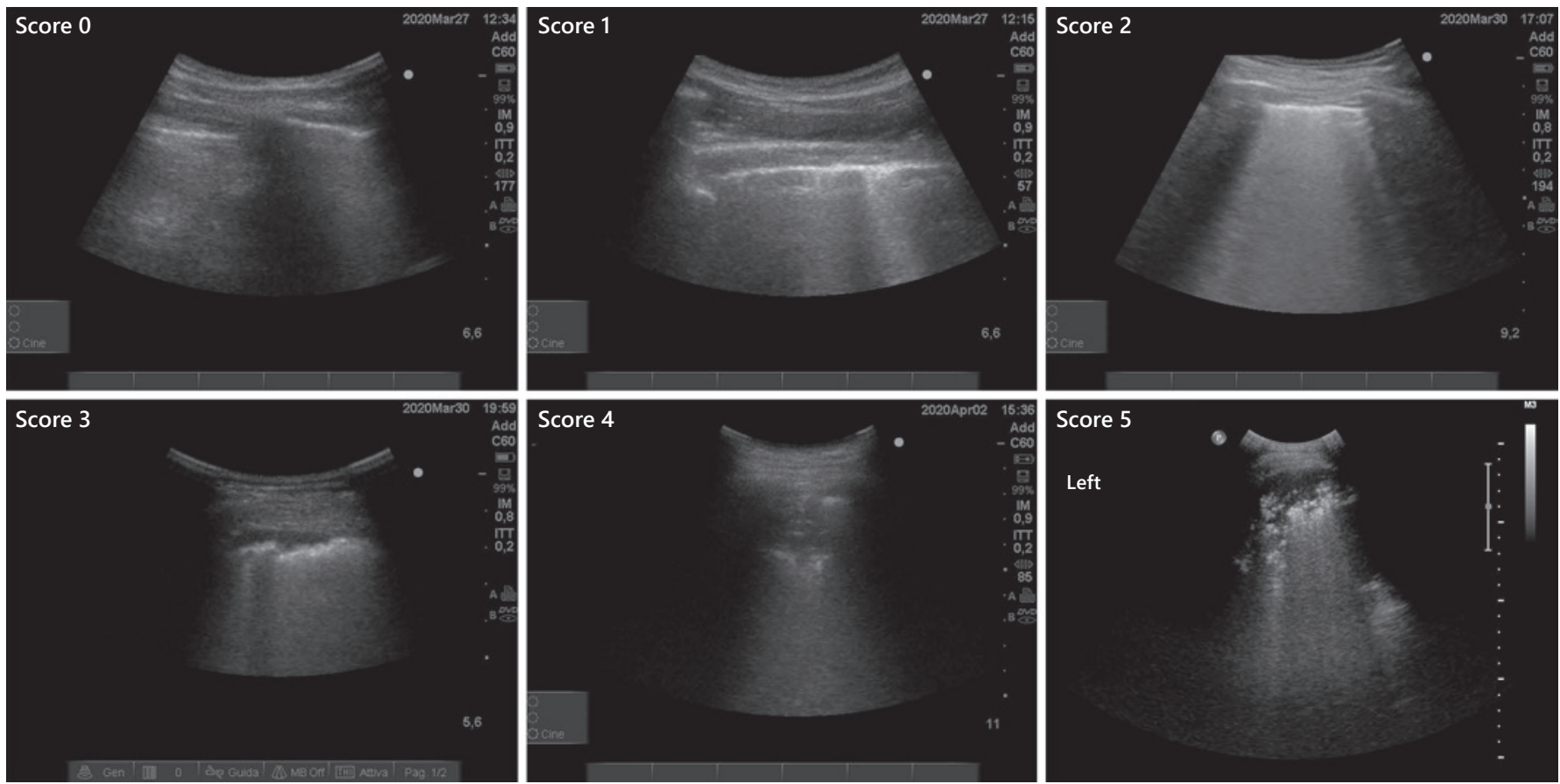

Fig. 2. LUS scoring. Score 0: normal lung surface with regular pleural line and no artifacts. Score 1: focal irregularities with vertical artifacts. Score 2: coalescent vertical artifact determining a focal white lung with mild surface irregularity. Score 3: subpleural con- solidation causing a marked surface irregularity with underlying white lung. Score 4: focal consolidation of $>2 \mathrm{~cm}$. Score 5: large consolidation with air bronchograms visible in more than one area. LUS, lung ultrasound. 


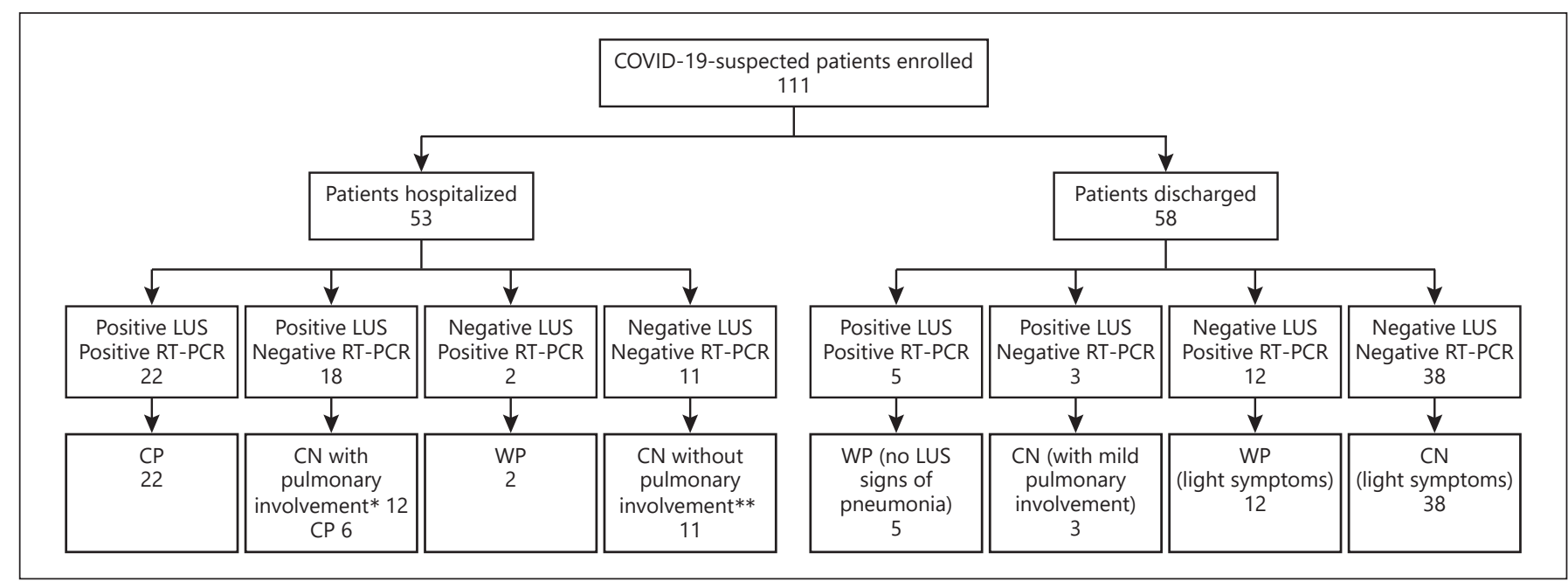

Fig. 3. Sample inclusion chart and outcome based on LUS and nasopharyngeal swab. * Congestive heart failure, carcinomatous lymphangitis, other viral pneumonia, bacterial pneumonia, pulmonary edema with renal failure, bilateral pleural effusions. ${ }^{* *}$ Urinary infections, exacerbation of ulcerative rectocolitis, esophageal

(Fig. 1). The severity score for each field was classified in five stages, from 0 (normal A pattern) to 5 (large consolidations extended to more fields) (Fig. 2). Operators were trained for the detection of lung fissures and not to mistake them for pathologic findings [25]. LUS operators worked wearing double gloves, FFP2 or FFP3 masks, whole protection suits, as well as protective face shields and footwear, and none of them became infected during the study. The LUS score was initially aimed at describing COVID-19 lung lesions and at recording them in the clinical documentation for a possible follow-up.

\section{Statistical Analysis}

Univariate contributions of predictors and group comparisons were assessed by means of the $\chi^{2}$ test for categorical data or the Kruskal-Wallis $\mathrm{H}$ test and the Mann-Whitney U test for continuous data. Therefore, we describe categorical data using frequencies or percentages, whereas means and standard deviations are given for continuous variables. To identify predictors that contribute independently to diagnosis, a stepwise logistic regression (forward Wald) was performed, and we computed predicted probabilities for each case. Receiver operating characteristic (ROC) curve analysis and the estimated area under the curve (AUC) were used to quantify how accurately LUS and nasopharyngeal swabs, alone or in combination with other parameters (predicted probabilities from logistic regression), could discriminate between $\mathrm{CP}$ and other diagnoses, and ROC was also used to visually assess an optimal cutoff. Furthermore, sensitivity and specificity were evaluated and we calculated respective CIs using the Jeffreys method [26]. All given $p$ values are two-sided and $p \leq 0.05$ was regarded as significant. SPSS IBM version 26 (IBM Corp., Armonk, NY, USA) was used for statistical analysis. varices with hematemesis, fever in chronic renal failure in dialysis, thoracic pain, legionellosis. CN, no coronavirus disease 2019; COVID-19, coronavirus disease 2019; CP, coronavirus disease 2019 pneumonia; LUS, lung ultrasound; RT-PCR, real-time polymerase chain reaction; WP, coronavirus disease 2019 without pneumonia.

\section{Results}

A total of 111 consecutive patients underwent ED evaluation with LUS assessment for suspected CP from March 24 to April 14, 2020 (Fig. 3) and were included in the study. The average duration of LUS examinations was 5-10 min.

Out of the 111 patients, 65 (59\%) were COVID-19-negative and 46 (41\%) were COVID-19-positive; 31 (67\%) of them were diagnosed with $\mathrm{CP}$ and 15 (33\%) had COVID-19 without pneumonia (WP). Demographics, clinical parameters, laboratory values, imaging features, and outcome variables are shown in Table 1 and Figure 3.

LUS pathological features in CP were mono- or bilateral, with distribution mainly in the posterior and lateral areas, consisting of pleural irregularities with increase in vertical artifacts, local and diffuse areas of "white lung" with surface irregularities, surface consolidations, and deeper confluent consolidations. No lobar or segmental distribution was observed as the lesions had often a patchy distribution. Pleural effusion was found only in 1 out of $31 \mathrm{CP}$ patients. The lowest LUS score in CP patients was 3 ( 2 cases) and the highest score was 41 (1 case), with a mean score of $11 \pm 10$ in CP patients compared to $3 \pm 6$ in WP patients and $3 \pm 6$ in COVID-19-negative patients (Kruskal-Wallis test, $p<0.001$ ).

Logistic regression analysis showed that the parameters fever (OR 6.5, 95\% CI 1.1-37.3, $p=0.035$ ), nasopha- 
Table 1. Descriptive statistics of the aggregated data of the entire population (111 patients), divided into CP, WP, and CN

\begin{tabular}{|c|c|c|c|c|c|}
\hline Variable & All $(n=111)$ & $\mathrm{CP}(n=31)$ & $\mathrm{WP}(n=15)$ & $\mathrm{CN}(n=65)$ & $p$ \\
\hline Sex, female/male & $54 / 57(49 / 52 \%)$ & $12 / 19(39 / 61 \%)$ & $8 / 7(53 / 47 \%)$ & $34 / 31(52 / 48 \%)$ & 0.430 \\
\hline Age, years & $55 \pm 21$ & $64 \pm 16$ & $51 \pm 18$ & $52 \pm 22$ & 0.019 \\
\hline Patients with comorbidities & $38(41 \%, 25-43 \%)$ & $7(23 \%, 11-39 \%)$ & $4(27 \%, 10-52 \%)$ & $27(41 \%, 30-54 \%)$ & 0.15 \\
\hline \multicolumn{6}{|l|}{ General symptoms } \\
\hline Cough & $69(63 \%, 54-72 \%)$ & $21(68 \%, 50-82 \%)$ & $8(53 \%, 29-76 \%)$ & $40(63 \%, 51-74 \%)$ & 0.63 \\
\hline Fever & $74(68 \%, 59-77 \%)$ & $28(90 \%, 76-97 \%)$ & $11(73 \%, 48-90 \%)$ & $35(56 \%, 44-68 \%)$ & 0.004 \\
\hline Dyspnea & $46(41 \%, 33-51 \%)$ & $13(42 \%, 26-60 \%)$ & $3(20 \%, 6-44 \%)$ & $30(48 \%, 36-60 \%)$ & 0.15 \\
\hline Weakness & $14(13 \%, 7-20 \%)$ & $5(16 \%, 6-31 \%)$ & $4(27 \%, 10-51 \%)$ & $5(8 \%, 3-16 \%)$ & 0.11 \\
\hline Thoracic pain & $12(11 \%, 6-18 \%)$ & 0 & $2(13 \%, 3-36 \%)$ & $10(16 \%, 8-26 \%)$ & 0.07 \\
\hline \multicolumn{6}{|l|}{ Neurological symptoms } \\
\hline Syncope & $2(2 \%, 1-6 \%)$ & $1(3 \%, 1-14 \%)$ & 0 & $1(2 \%, 1-7 \%)$ & \multirow{3}{*}{0.54} \\
\hline Smell/taste loss & $6(5 \%, 2-11 \%)$ & $2(7 \%, 1-20 \%)$ & $2(13 \%, 3-36 \%)$ & $2(3 \%, 1-10 \%)$ & \\
\hline Headache & $4(4 \%, 2-9 \%)$ & $1(3 \%, 1-14 \%)$ & 0 & $3(5 \%, 1-12 \%)$ & \\
\hline \multicolumn{6}{|l|}{ Gastroenteric symptoms } \\
\hline Vomiting & $5(5 \%, 2-10 \%)$ & $2(7 \%, 1-20 \%)$ & 0 & $3(5 \%, 1-7 \%)$ & \multirow{2}{*}{0.84} \\
\hline Diarrhea & $6(5 \%, 2-11 \%)$ & $4(13 \%, 5-29 \%)$ & $2(13 \%, 3-36 \%)$ & $6(9 \%, 4-19 \%)$ & \\
\hline \multicolumn{6}{|l|}{ Others uncommon symptoms } \\
\hline Confusion & $2(2 \%, 1-6 \%)$ & $2(7 \%, 1-20 \%)$ & 0 & 0 & \multirow{3}{*}{0.43} \\
\hline Paresthesia & $1(1 \%, 1-4 \%)$ & 0 & $1(7 \%, 1-27 \%)$ & 0 & \\
\hline Nasal obstruction & $1(1 \%, 1-4 \%)$ & 0 & 0 & $1(2 \%, 1-7 \%)$ & \\
\hline \multicolumn{6}{|c|}{ Other non-COVID-19-related symptoms } \\
\hline Leg edemas & $4(4 \%, 2-9 \%)$ & 0 & 0 & $4(7 \%, 2-14 \%)$ & \multirow{4}{*}{0.87} \\
\hline Abdominal pain & $1(1 \%, 1-4 \%)$ & $1(3 \%, 1-14 \%)$ & 0 & 0 & \\
\hline Leg pain & $1(1 \%, 1-4 \%)$ & 0 & 0 & $1(2 \%, 1-7 \%)$ & \\
\hline Hemoptysis & $1(1 \%, 1-4 \%)$ & & 0 & $1(2 \%, 1-7 \%)$ & \\
\hline $\mathrm{SpO}_{2}, \%$ & $96 \pm 3$ & $94 \pm 3$ & $97 \pm 2$ & $96 \pm 3$ & 0.000 \\
\hline \multicolumn{6}{|l|}{ Blood gas analysis } \\
\hline $\mathrm{PaO}_{2}, \mathrm{~mm} \mathrm{Hg}$ & $79 \pm 18$ & $68 \pm 15$ & $83 \pm 23$ & $84 \pm 16$ & \multirow{2}{*}{0.000} \\
\hline $\mathrm{PaO}_{2} / \mathrm{FiO}_{2}$ & $376 \pm 87$ & $323 \pm 74$ & $394 \pm 110$ & $398 \pm 77$ & \\
\hline Lymphopenia $(<1,100 / \mu \mathrm{L})$ & $36(32 \%, 24-42 \%)$ & $18(58 \%, 41-74 \%)$ & $3(20 \%, 6-44 \%)$ & $15(23 \%, 14-35 \%)$ & 0.002 \\
\hline Increased LDH (>230 U/L) & $49(46 \%, 37-56 \%)$ & $22(71 \%, 54-85 \%)$ & $4(29 \%, 10-54 \%)$ & $23(48 \%, 26-50 \%)$ & 0.004 \\
\hline $\mathrm{CRP}, \mathrm{mg} / \mathrm{dL}$ & $3.1 \pm 4.9$ & $4.28 \pm 3.61$ & $0.81 \pm 1.16$ & $3.1 \pm 5.8$ & 0.000 \\
\hline \multicolumn{6}{|l|}{ Other laboratory values } \\
\hline NT-proBNP $(>125$ pg/mL) & $11(10 \%, 5-16 \%)$ & $1(3 \%, 1-14 \%)$ & 0 & $10(16 \%, 8-26 \%)$ & \multirow{4}{*}{0.16} \\
\hline Troponin-T (14 ng/L) & $5(5 \%, 2-10 \%)$ & $1(3 \%, 1-14 \%)$ & 0 & $4(7 \%, 2-14 \%)$ & \\
\hline Procalcitonin $(>0.05 \mathrm{ng} / \mathrm{mL})$ & $12(11 \%, 6-18 \%)$ & $3(10 \%, 2-24 \%)$ & $1(7 \%, 1-27 \%)$ & $8(12 \%, 6-23 \%)$ & \\
\hline D-dimer $(500 \mathrm{ng} / \mathrm{mL})$ & $3(3 \%, 1-7 \%)$ & 0 & 0 & $3(5 \%, 1-12 \%)$ & \\
\hline \multicolumn{6}{|l|}{ Chest X-ray, $n /$ total } \\
\hline Positive $\mathrm{e}^{1}$ & $33(30 \%, 22-39 \%)$ & $19(63 \%, 45-79 \%)$ & $2(14 \%, 3-38 \%)$ & $12(18 \%, 10-29 \%)$ & \multirow{3}{*}{0.000} \\
\hline Negative & $74(68 \%, 59-76 \%)$ & $10(33 \%, 19-51 \%)$ & $12(86 \%, 61-97 \%)$ & $52(80 \%, 69-88 \%)$ & \\
\hline Uncertain & $2(2 \%, 1-6 \%)$ & $1(3 \%, 1-15 \%)$ & 0 & $1(2 \%, 1-7 \%)$ & \\
\hline \multicolumn{6}{|l|}{ LUS } \\
\hline Positive $^{1}$ & $33(30 \%, 22-39 \%)$ & $25(81 \%, 64-91 \%)$ & $2(13 \%, 3-36 \%)$ & $6(9 \%, 4-18 \%)$ & \multirow{4}{*}{0.000} \\
\hline Negative & $62(56 \%, 47-65 \%)$ & $4(13 \%, 5-28 \%)$ & $13(87 \%, 64-97 \%)$ & $45(69 \%, 57-79 \%)$ & \\
\hline Uncertain & $16(14 \%, 9-22 \%)$ & $2(7 \%, 1-19 \%)$ & 0 & $14(21 \%, 13-33 \%)$ & \\
\hline Mean score & $5.1 \pm 7.8$ & $11.1 \pm 9.6$ & $1.5 \pm 2.8$ & $2.9 \pm 5.8$ & \\
\hline \multicolumn{6}{|l|}{ Chest CT } \\
\hline Performed in & $14(13 \%, 7-20 \%)$ & $5(16 \%, 6-32 \%)$ & $1(7 \%, 1-27 \%)$ & $8(12 \%, 6-23 \%)$ & \multirow{4}{*}{0.002} \\
\hline Positive $^{1}$ & $6(5 \%, 2-11 \%)$ & $5(16 \%, 6-32 \%)$ & 0 & $1(2 \%, 1-7 \%)$ & \\
\hline Negative & $7(6 \%, 3-12 \%)$ & 0 & 0 & $7(11 \%, 5-20 \%)$ & \\
\hline Uncertain & $1(1 \%, 1-4 \%)$ & 0 & $1(7 \%, 1-27 \%)$ & 0 & \\
\hline
\end{tabular}


Table 1 (continued)

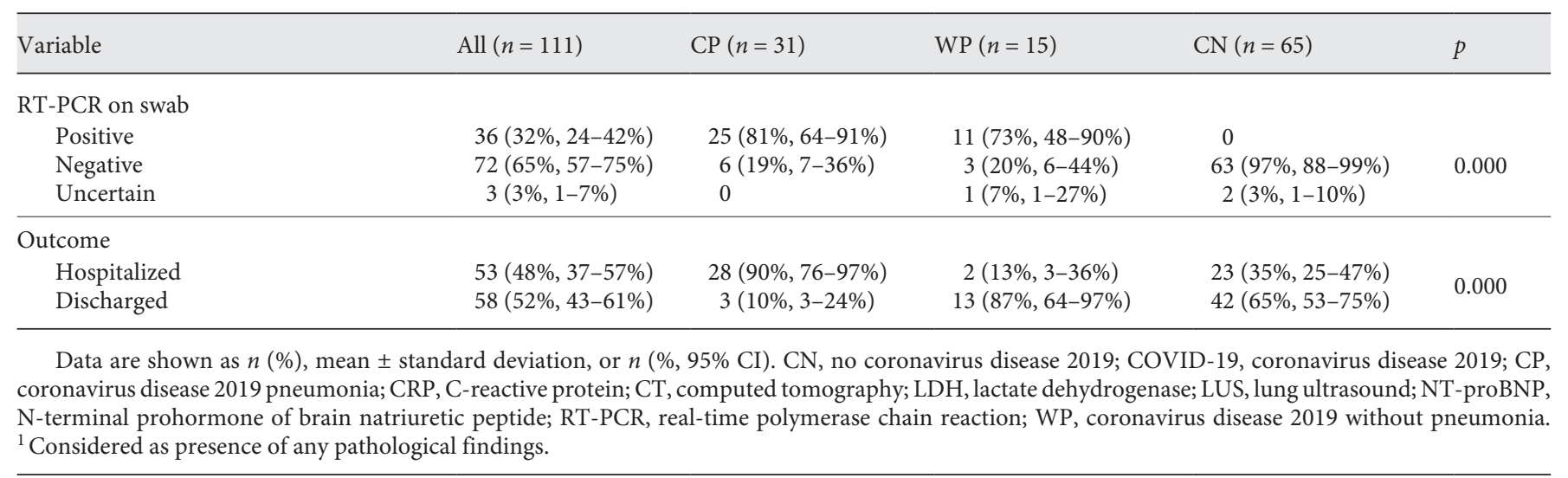

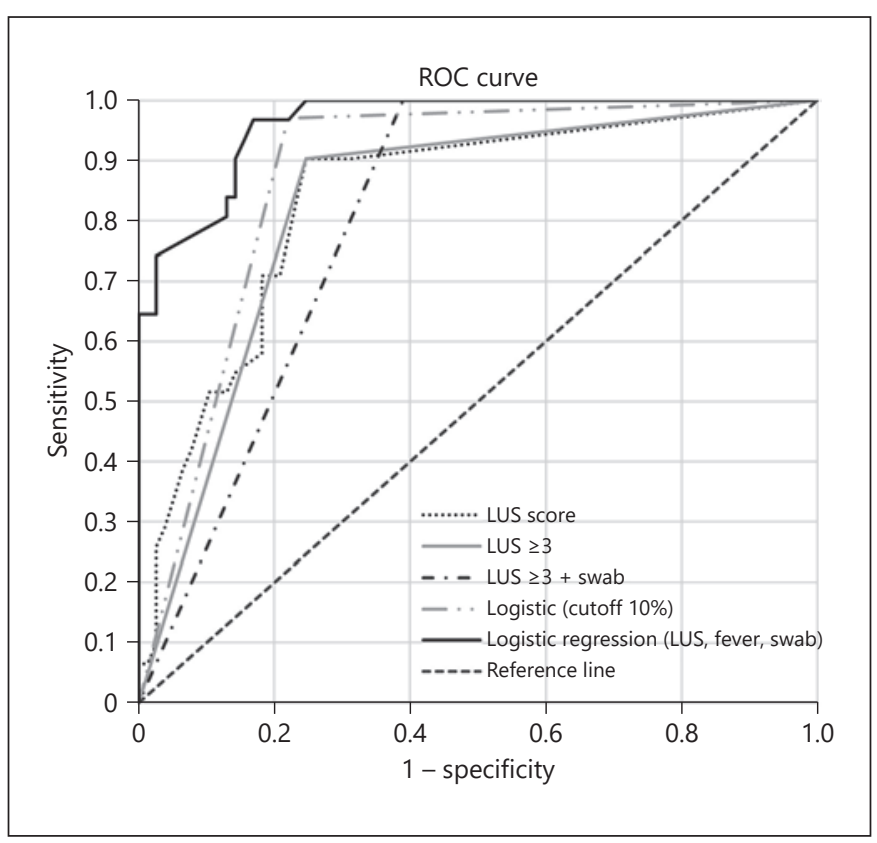

Fig. 4. ROC-AUC for CP diagnosis. Shown are ROC curves for the LUS score with an AUC of 0.84 (95\% CI 0.75-0.92), applying the cutoff LUS $\geq 3$ with an AUC of 0.83 (95\% CI 0.74-0.91), combining LUS $\geq 3$ with a positive or inconclusive swab with an AUC of 0.81 (95\% CI 0.73-0.88), calculated probabilities from logistic regression using LUS score, fever, and swab (positive, inconclusive) with an AUC of 0.96 (95\% CI 0.93-0.99), and applying the cutoff of $10 \%$ to calculated probabilities from logistic regression with an AUC of 0.87 (95\% CI 0.81-0.94). AUC, area under the curve; CP, coronavirus disease 2019 pneumonia; LUS, lung ultrasound; ROC, receiver operating characteristic; ROC-AUC, area under the corresponding receiver operating characteristic curve. ryngeal swab (OR 27, 95\% CI 6-122, $p<0.001$ ), and LUS score (OR 1.3 per unit, 95\% CI 1.1-1.5, $p<0.001$ ) were independently related to $\mathrm{CP}$ diagnosis.

The area under the corresponding ROC curve (ROCAUC) for CP diagnosis using nasopharyngeal swab (positive or inconclusive) was 0.80 (95\% CI 0.70-0.90) (Fig. 4) and accurately identified 25 of $31 \mathrm{CP}$ patients and 14 of 15 WP patients (sensitivity $81 \%$, 95\% CI 74-97\%; specificity $80 \%, 95 \%$ CI $44-67 \%)$. The ROC-AUC of LUS score was 0.84 (95\% CI 0.75-0.92) and, when using a cutoff value $\geq 3$, accurately identified 28 of 31 patients with $\mathrm{CP}$ and 11 of 15 with WP (sensitivity 90\%, 95\% CI 74-97\%; specificity $75 \%, 95 \%$ CI $56-76 \%)$. A ROC-AUC of LUS in combination with nasopharyngeal swab (one of both positive) of 0.81 (95\% CI $0.73-0.89)$ accurately identified all 31 patients with $\mathrm{CP}$ and 14 of $15 \mathrm{WP}$ patients (sensitivity 100\%, 95\% CI 74-97\%; specificity 61\%, 95\% CI 44-67\%). The ROC-AUC using the predicted probabilities from logistic regression with the parameters LUS score, swabs, and fever was 0.96 (95\% CI 0.93-0.99) and, when using a cutoff of $10 \%$, accurately identified 30 of 31 patients with $\mathrm{CP}$ and 12 of 15 patients with WP (sensitivity $97 \%, 95 \%$ CI 74-97\%; specificity 78\%, 95\% CI 44-67\%).

Using a LUS score $\geq 3$, we identified 40 out of 53 (76\%) patients who needed hospital admission and 50 out of 58 (87\%) who did not (Fig. 3; Table 2). From the total of 48 LUS-positive patients, 27 (57\%) had a positive nasopharyngeal swab, of whom $22(81 \%)$ had been hospitalized. LUS was positive in $18(86 \%)$ out of the $21(43 \%)$ patients with a negative swab; within 6 cases, a first swab was negative but LUS suggested CP; conversely, LUS was positive and swab negative in cases with a diagnosis other than $\mathrm{CP}$ requiring hospital admission (Fig. 3). Eight patients with positive LUS were discharged because hospital admission 
Table 2. Descriptive statistics of the aggregated data of the entire population (111 patients), divided into patient outcome (discharged/hospitalized) based on LUS score and RT-PCR assay of nasopharyngeal swab

\begin{tabular}{|c|c|c|c|c|c|c|}
\hline \multirow{2}{*}{$\begin{array}{l}\text { LUS score } \\
\text { RT-PCR assay }\end{array}$} & \multicolumn{3}{|l|}{$<3$} & \multicolumn{3}{|l|}{$\geq 3$} \\
\hline & negative & positive & total & negative & positive & total \\
\hline Discharged & $38(60.4 \%)$ & $12(19 \%)$ & $50(79.4 \%)$ & $3(6.3 \%)$ & $5(10.4 \%)$ & $8(16.7 \%)$ \\
\hline Hospitalized & $11(17.4 \%)$ & $2(3.2 \%)$ & $13(20.6 \%)$ & $18(37.5 \%)$ & $22(45.8 \%)$ & $40(83.3 \%)$ \\
\hline Total & $49(77.8 \%)$ & $14(22.2 \%)$ & $63(100 \%)$ & $21(43.8 \%)$ & $27(56.2 \%)$ & $48(100 \%)$ \\
\hline
\end{tabular}

Data are shown as $n(\%)$. LUS, lung ultrasound; RT-PCR, real-time polymerase chain reaction.

was clinically not required, although 5 of them had a positive swab. Patients with a LUS score $\geq 3$ had lower $\mathrm{SpO}_{2}$ values (median 95 vs. 97\%, $p<0.001$ ), lower $\mathrm{pO}_{2}$ (median 69 vs. $88 \mathrm{~mm} \mathrm{Hg}, p<0.001$ ), and lower $\mathrm{pO}_{2} / \mathrm{FiO}_{2}$ (median 323 vs. $413, p<0.001)$.

\section{Discussion}

LUS seems to be a promising technique for early identification of CP in patients with suspected COVID-19 accessing the ED in an active epidemic time. The LUS score shows a sensitivity of $90 \%$ for $\mathrm{CP}$, allowing to quickly refer patients with COVID-19 to the ED isolation area or to discharge them for outpatient treatment.

LUS in CP showed a distribution of pathological features mainly involving both lungs, with multiple lung lobes and segments affected, similarly to the pattern already described [15]. These patterns are consistent with CT features $[15,27,28]$. The retrospective analysis allowed us to identify a cutoff value of the LUS score, and ROC curve analysis of LUS score $\geq 3$ identified patients with CP with a sensitivity and specificity of 90 and $75 \%$, respectively. The low cutoff value of the score means that minimum findings (i.e., two focal areas of "white lung" with a score of $2+2$ ) can be diagnostic for CP. Such a low value of the score suggests that LUS may really improve early diagnosis of pneumonia and ED management strategies. Specifically, during an acute epidemic, in presence of organizational difficulties in performing CT scans, LUS could allow early diagnosis of $\mathrm{CP}$, limiting the risk of spreading the virus when adequate protective paths are still not available. Nevertheless, further prospective studies are needed to validate this LUS score cutoff value.

LUS features are not specific for CP, as LUS acts like a lung densitometry. LUS interstitial syndrome gives infor- mation about a surface dysventilation of the lung, and a consolidative pattern indicates the areas of major air loss $[29,30]$. These patterns can also be found in many different lung diseases like other viral or bacterial pneumonias, carcinomatous lymphangitis, interstitial lung diseases, and lung contusions, but when LUS features and topographic distribution are combined with clinical and laboratory data, the diagnosis may be reliable. The combination of LUS, fever, and nasopharyngeal swab showed $97 \%$ sensitivity and $78 \%$ specificity in identifying patients with $\mathrm{CP}$, suggesting a possible simplified diagnostic strategy with limited costs and time. Conversely, the combination of LUS and nasopharyngeal swab showed $100 \%$ sensitivity and $61 \%$ specificity in identifying patients with CP. Such combination allowed to correctly manage patients triaged for hospital admission.

Patients with CP in the ED were older males with fever and cough and a higher prevalence of lymphopenia, increased lactate dehydrogenase and C-reactive protein, impaired $A B G$ values, and abnormal imaging features compared to WP patients or a diagnosis other than COVID-19. These findings are consistent with those of other recent studies [31, 32] and, compared to previous studies reporting CT findings and LUS findings of CP $[15,27]$, we describe imaging findings in combination with symptoms and laboratory data. Specifically, a linear regression between LUS score and $\mathrm{SpO}_{2}, \mathrm{pO}_{2}$, and $\mathrm{pO}_{2} / \mathrm{FiO}_{2}$ suggests a significant correlation between increasing LUS score and worsening $\mathrm{O}_{2}$ exchanges. Even if LUS assessment is limited to lung surface and does not allow the evaluation of deep parenchyma, such findings further support the already reported consistency in CP between LUS and CT scan [15]. Although our aim was not to stratify CP severity, our data suggest the evaluation of such application of LUS in further studies and its usefulness also to establish the correct level of respiratory support [33]. 
Outpatient follow-up of COVID-19 patients without imaging features of pneumonia and with absence of other "red flags" is being implemented by general practitioners and infection control and prevention units [34, 35]. Primary care providers must be informed, and patients must be both instructed and motivated to follow the quarantine measures and react in case of clinical worsening. LUS was very useful to exclude pulmonary involvement early: this allowed early discharge of patients without pneumonia and still waiting for the RT-PCR result. With such a management strategy, the workload of the ED was reduced and dedicated to patients waiting to be addressed to COVID or not-COVID wards based on their RT-PCR result, and those needing more attention and assistance.

Our study has some limitations. First, our data should be contextualized in an active epidemic time (e.g., March to April 2020 in Italy, when the high number of subjects suspected to be infected increased the pretest probability), and further studies are needed to investigate its usefulness for the diagnosis of CP as the epidemic curve is decreasing. Second, as it was not possible to guarantee complete covering of medical wards with LUS-trained personnel, our data are based on cases consecutively recruited only in LUS-powered sessions. Multicenter studies are needed for a more precise definition of sensitivity and specificity of LUS in CP. Finally, as the patients were directed to different wards and hospitals, only the ED documentation was included in the analysis, so a correlation between LUS pattern and outcome was not possible. Nevertheless, we found a correlation between increasing LUS score and worsening hypoxemia.

LUS is a promising technique for early identification of $\mathrm{CP}$ in patients who access the ED with suspicion of COVID-19. Standardization of the LUS technique and further prospective multicenter studies, even in other pandemic phases, can help improve the reliability of LUS and the proposed cutoff score for its use for early diagnosis of CP during a pandemic.

\section{Acknowledgments}

We thank the personnel of the South Tyrolean Health Care System, Italy, for their relentless efforts and invaluable work during the COVID-19 pandemic.

\section{Statement of Ethics}

This study was conducted in accordance with the amended Declaration of Helsinki. It was approved by the local institutional review board (BZ 34-2020). Informed consent was not required.

\section{Conflict of Interest Statement}

No conflict of interest exists for any of the authors.

\section{Funding Sources}

None.

\section{Author Contributions}

A. Zanforlin takes responsibility for the content of the manuscript and data analysis. A. Zanforlin, G. Strapazzon, M. Falk, N. Vezzali: conceptualization. A. Zanforlin, G. Strapazzon, F. Ferro, N. Vezzali: project administration and supervision. A. Zanforlin, V. Gallina, L. Pagani, A. Viteritti, L. Valzolgher, M. La Guardia, F. Ferro, N. Vezzali: methodology. A. Zanforlin, V. Gallina, L. Pagani, A. Viteritti, L. Valzolgher, M. La Guardia, F. Ferro, N. Vezzali: investigation. A. Zanforlin, G. Strapazzon, M. Falk: data analysis and interpretation. A. Zanforlin, G. Strapazzon, M. Falk, L. Pagani: original draft. A. Zanforlin, G. Strapazzon, M. Falk, V. Gallina, L. Pagani, A. Viteritti, L. Valzolgher, M. La Guardia, F. Ferro, N. Vezzali: review and editing.

\section{References}

1 World Health Organization. Coronavirus disease (COVID-19) pandemic. www.who. $\mathrm{int} /$ emergencies/diseases/novel-coronavirus-2019 [accessed May 4, 2020].

2 Italian Thoracic Society. Approccio pragmatico alla diagnosi di polmonite da SARSCoV-2 [COVID-19]. www.aiponet.it/component/attachments/download/2647.html [accessed May 4, 2020].
3 Barton LM, Duval EJ, Stroberg E, Ghosh S, Mukhopadhyay S. COVID-19 autopsies, Oklahoma, USA. Am J Clin Pathol. 2020 May; 153(6):725-33.

4 Pan F, Ye T, Sun P, Gui S, Liang B, Li L, et al. Time Course of Lung Changes at Chest CT during Recovery from Coronavirus Disease 2019 (COVID-19). Radiology. 2020 Jun; 295(3):715-21.
5 Peng QY, Wang XT, Zhang LN; Chinese Critical Care Ultrasound Study Group (CCUSG). Findings of lung ultrasonography of novel corona virus pneumonia during the 2019-2020 epidemic. Intensive Care Med. 2020 May; 46(5):849-50.

6 Nouvenne A, Zani MD, Milanese G, Parise A, Baciarello M, Bignami EG, et al. Lung ultrasound in COVID-19 pneumonia: correlations with chest $\mathrm{CT}$ on hospital admission. Respiration. 2020;99(7):617-24. 
7 Shaw JA, Louw EH, Koegelenberg CF. Lung Ultrasound in COVID-19: Not Novel, but Necessary. Respiration. 2020;99(7):545-7.

8 Zanforlin A, Tursi F, Marchetti G, Pellegrino GM, Vigo B, Smargiassi A, et al.; AdET Study Group. Clinical Use and Barriers of Thoracic Ultrasound: A Survey of Italian Pulmonologists. Respiration. 2020;99(2):171-6.

9 Accademia di Ecografia Toracica. www.facebook.com/groups/1118364641543554/ [accessed May 4, 2020].

10 Wang L, Song W, Wang Y, Han J, Lv K. Lung ultrasonography versus chest radiography for the diagnosis of pediatric community acquired pneumonia in emergency department: a meta-analysis. J Thorac Dis. 2019 Dec; 11(12):5107-14.

11 Orso D, Guglielmo N, Copetti R. Lung ultrasound in diagnosing pneumonia in the emergency department: a systematic review and meta-analysis. Eur J Emerg Med. 2018 Oct: 25(5):312-21.

12 Alzahrani SA, Al-Salamah MA, Al-Madan WH, Elbarbary MA. Systematic review and meta-analysis for the use of ultrasound versus radiology in diagnosing of pneumonia. Crit Ultrasound J. 2017 Dec;9(1):6.

13 Xia Y, Ying Y, Wang S, Li W, Shen H. Effectiveness of lung ultrasonography for diagnosis of pneumonia in adults: a systematic review and meta-analysis. J Thorac Dis. 2016 Oct; $8(10): 2822-31$.

14 Llamas-Álvarez AM, Tenza-Lozano EM, Latour-Pérez J. Accuracy of Lung Ultrasonography in the Diagnosis of Pneumonia in Adults: Systematic Review and Meta-Analysis. Chest. 2017 Feb;151(2):374-82.

15 Lu W, Zhang S, Chen B, Chen J, Xian J, Lin Y, et al. A clinical study of noninvasive assessment of lung lesions in patients with coronavirus disease-19 (COVID-19) by bedside ultrasound. Ultraschall Med. 2020 Jun;41(3): 300-7.

16 Soldati G, Smargiassi A, Inchingolo R, Buonsenso D, Perrone T, Briganti DF, et al. Proposal for international standardization of the use of lung ultrasound for patients with $\mathrm{CO}$ VID-19: a simple, quantitative, reproducible method. J Ultrasound Med. 2020 Jul;39(7) $1413-9$.
17 Buonsenso D, Pata D, Chiaretti A. COVID-19 outbreak: less stethoscope, more ultrasound. Lancet Respir Med. 2020 May;8(5):e27.

18 Buonsenso D, Piano A, Raffaelli F, Bonadia N, de Gaetano Donati K, Franceschi F. Point-ofcare lung ultrasound findings in novel coronavirus disease-19 pneumonia: a case report and potential applications during COVID-19 outbreak. Eur Rev Med Pharmacol Sci. 2020 Mar;24(5):2776-80.

19 Soldati G, Smargiassi A, Inchingolo R, Buonsenso $\mathrm{D}$, Perrone $\mathrm{T}$, Briganti $\mathrm{DF}$, et al. Is there a role for lung ultrasound during the $\mathrm{CO}$ VID-19 pandemic? J Ultrasound Med. 2020 Jul;39(7):1459-62.

20 European Federation of Societies for Ultrasound in Medicine and Biology. Ultrasound evaluation of COVID pneumonia. www. you tube.com/watch ? v = c T Z zrB o5iI\&feature=youtu.be [accessed May 4, 2020].

21 Carenzo L, Costantini E, Greco M, Barra FL, Rendiniello V, Mainetti M, et al. Hospital surge capacity in a tertiary emergency referral centre during the COVID-19 outbreak in Italy. Anaesthesia. 2020 Jul;75(7):928-34.

22 Sethuraman N, Jeremiah SS, Ryo A. Interpreting Diagnostic Tests for SARS-CoV-2. JAMA. 2020 Jun;323(22):2249-51.

23 Istituto Superiore di Sanità. Raccomandazioni ad interim per il corretto prelievo, conservazione e analisi sul tampone rino/orofaringeo per la diagnosi di COVID-19. www. epicentro.iss.it/coronavirus/pdf/rapportocovid-19-11-2020.pdf [accessed May 4, 2020].

24 Bouhemad B, Mongodi S, Via G, Rouquette I. Ultrasound for "lung monitoring" of ventilated patients. Anesthesiology. 2015 Feb; 122(2):437-47.

25 Zanforlin A, Livi V, Santoriello C, Ceruti P, Trigiani M, Valerio M, et al. Ultrasound Fissure Observation: Assessment of Lung by Pleural-Hub Affiliates. Chest. 2018 Aug; 154(2):357-62.
26 Brown LD, Cat TT, DasGupta A. Interval estimation for a proportion. Stat Sci. 2001; 16(2):101-33.

27 Shi H, Han X, Jiang N, Cao Y, Alwalid O, Gu $\mathrm{J}$, et al. Radiological findings from 81 patients with COVID-19 pneumonia in Wuhan, China: a descriptive study. Lancet Infect Dis. 2020 Apr;20(4):425-34.

28 Chung M, Bernheim A, Mei X, Zhang N, Huang $M$, Zeng $X$, et al. CT imaging features of 2019 novel coronavirus (2019-nCoV). Radiology. 2020 Apr;295(1):202-7.

29 Soldati G, Smargiassi A, Inchingolo R, Sher S, Nenna R, Valente S, et al. Lung ultrasonography may provide an indirect estimation of lung porosity and airspace geometry. Respiration. 2014;88(6):458-68.

30 Soldati G, Inchingolo R, Smargiassi A, Sher S, Nenna R, Inchingolo CD, et al. Ex vivo lung sonography: morphologic-ultrasound relationship. Ultrasound Med Biol. 2012 Jul; 38(7):1169-79.

31 Guan WJ, Ni ZY, Hu Y, Liang WH, Ou CQ, $\mathrm{He}$ JX, et al.; China Medical Treatment Expert Group for Covid-19. Clinical Characteristics of Coronavirus Disease 2019 in China. N Engl J Med. 2020 Apr;382(18):1708-20.

32 Chen N, Zhou M, Dong X, Qu J, Gong F, Han $\mathrm{Y}$, et al. Epidemiological and clinical characteristics of 99 cases of 2019 novel coronavirus pneumonia in Wuhan, China: a descriptive study. Lancet. 2020 Feb;395(10223):507-13.

33 Italian Thoracic Society. Managing the respiratory care of patients with COVID-19. www. aiponet.it/component/attachments/download/2626.html [accessed May 4, 2020].

34 Medina M, Babiuch C, Card M, Gavrilescu R, Zafirau W, Boose E, et al. Home monitoring for COVID-19. Cleve Clin J Med. doi: 10.3949/ccjm.87a.ccc028 [Epub ahead of print].

35 Annis T, Pleasants S, Hultman G, Lindemann E, Thompson JA, Billecke S, et al. Rapid implementation of a COVID-19 remote patient monitoring program. J Am Med Inform Assoc. 2020 Aug;27(8):1326-30. 